\title{
Parasitoses intestinais de indígenas da comunidade Mapuera (Oriximiná, Estado do Pará, Brasil): elevada prevalência de Blastocystis bominis e encontro de Cryptosporidium sp e Cyclospora cayetanensis
}

\author{
Intestinal parasitosis in Indians of the Mapuera community (Oriximiná, \\ State of Pará, Brazil): high prevalence of Blastocystis hominis and finding of \\ Cryptosporidium sp and Cyclospora cayetanensis
}

\author{
Jaila Dias Borges ${ }^{1}$, Ruth Semira Rodríguez Alarcón², \\ Vicente Amato Neto ${ }^{2}$ e Erika Gakiya ${ }^{2}$
}

\begin{abstract}
RESUMO
Foi avaliada a ocorrência de parasitoses intestinais em indígenas da aldeia Mapuera (Oriximiná, Estado do Pará, Brasil). No contexto de apreciações congêneres, expressa contribuição para adequado conhecimento do assunto, significativo sob o ponto de vista médico-sanitário. 0 exame parasitológico das fezes, de 83 pessoas, realizado por meio de quatro métodos, pode ser considerado como dotado de razoável amplitude para estabelecer diagnósticos. Ocorreu encontro de cistos de protozoários e de ovos de helmintos de múltiplos tipos, até mesmo em expressivas porcentagens, merecendo destaque a muito frequiente presença de Blastocystis hominis (57,8\%), como também o encontro de Cryptosporidium sp (3,6\%) e de Cyclospora cayetanensis $(10,8 \%)$, comentado especificamente. 0 verificado demonstra que tais índios vivem em ambiente onde prevalecem más condições higiênicas, em especial, facilitador da disseminação de protozoários e helmintos pelo contato com o solo ou ingestão de água e alimentos contaminados.
\end{abstract}

Palavras-chaves: Enteroparasitoses. Indígenas (Mapuera, Estado do Pará).

\section{ABSTRACT}

Occurrences of intestinal parasitosis in Indians of the Mapuera community (Oriximiná, State of Pará, Brazil) were evaluated. Within the context of group assessment, this study makes a contribution towards adequate knowledge of this subject, which is significant from a medical-sanitary point of view. Parasitological examination of feces from 83 individuals, performed using four different methods, could be considered to have reasonable amplitude for establishing diagnoses. Protozoan cysts and helminth eggs of many types were found, even with significant percentages. The frequent presence of Blastocystis hominis (57.8\%), along with findings of Cryptosporidium sp (3.6\%) and Cyclospora cayetanensis (10.8\%), deserved highlighting with specific comments. The findings show that these Indians live in an environment in which poor hygiene conditions prevail. In particular, these facilitate the dissemination of protozoa and helminths through contact with the soil or through intake of contaminated water and food.

Key-words: Enteroparasitosis. Indians (Mapuera, State of Pará).

As fezes foram trazidas por pesquisadora que esteve na aldeia para realizar determinada investigação científica.

Para conhecer particularidades concernentes a indígenas, no Brasil, várias apreciações já foram ou ainda são realizadas. Diversas publicações atestam isso e, como exemplos, abordaram diabetes, alcoolismo, estado nutricional, segurança alimentar, questão sociolingüística, epidemiologia, câncer e nutrição no âmbito da saúde infantil ${ }^{8}$. Parasitoses intestinais ficaram reiteradamente focalizadas e demonstraram percalços expressivos

1.Laboratório de Virologia do Instituto de Medicina Tropical de São Paulo, SP. 2.Laboratório de Investigação Médica-Parasitologia do Hospital das Clínicas, da Faculdade de Medicina da Universidade de São Paulo, SP.

Endereço para correspondência: Prof. Vicente Amato Neto. Av. Dr. Enéas de Carvalho Aguiar 500, 05403-000 São Paulo, SP.

e-mail: amatonet@usp.br

Recebido para publicação em 18/08/2008

Aceito em 29/04/2009 em termos de más condições sanitárias e enfermidades ${ }^{710}$. Conforme explicamos antes aproveitamos a ocasião para executar algo congênere em mais um grupo de índios. Relataremos nossas verificações, que destacam a elevada presença de Blastocystis bominis e a participação de Cryptosporidium sp e Cyclospora cayetanensis.

A comunidade vive em margem do rio Mapuera, na região noroeste do Município de Oriximiná (Estado do Pará). É formada por dez aldeias, constituídas de taipa não revestida, e tem 987 habitantes, contados os adultos e as crianças. Falam o Wai-Wai, língua nativa e dependem da agricultura, da pesca e do artesanato para subsistência. A alimentação tem como base farinha de mandioca, beju, peixe, frutas e carne de animais caçados. Os excretas são eliminados em fossas e o lixo é enterrado. Consomem a água obtida diretamente do rio. Há luz elétrica durante parte do dia, escola para educação de alunos até a sexta 
série e posto de saúde onde exercem atividades enfermeira, auxiliar de enfermagem, além de dentista, mas não existe atendimento médico. 0 panorama geral atesta a vigência de claras inadequações a respeito da presença de circunstâncias favoráveis à aquisição de enteroparasitoses, constituídas mormente por má qualidade de higiene e hábitos impróprios.

Em agosto de 2006, recebemos 83 amostras de fezes de homens ou mulheres com até sessenta anos de idade. Estavam conservadas em formol a 10\%. Para os exames usamos os seguintes métodos: direto; centrífugo-flutuação em sulfato de zinco; sedimentação espontânea em água; coloração para coccídeos ${ }^{14}$ estão especificados na Tabela 1.

\section{TABELA 1}

Protozoários e helmintos revelados pelo exame parasitológico de fezes de 83 indígenas da aldeia Mapuera (Oriximiná, Estado do Pará, Brasil).

\begin{tabular}{lcc}
\hline Protozoários & Número & Porcentagem \\
\hline Entamoeba coli* & 49 & 59,0 \\
Blastocystis hominis & 48 & 57,8 \\
Endolimax nana* & 40 & 38,2 \\
Entamoeba bistolytica/dispar & 36 & 43,4 \\
Iodamoeba bütschlii* & \\
Chilomastix mesnili* & 27 & 32,5 \\
Giardia lamblia & 22 & 26,5 \\
Cyclospora cayetanensis & 10 & 12,0 \\
Cryptosporidium sp & 9 & 10,8 \\
Helmintos & 3 & 3,6 \\
Hymenolepis nana & & \\
Ascaris lumbricoides & 17 & 20,5 \\
Enterobius vermicularis & 6 & 7,2 \\
\hline
\end{tabular}

*comensais.

As porcentagens de protozoários e helmintos encontradas são relevantes. Dependem acima de tudo do contato não protegido com o solo e da ingestão de água ou alimentos contaminados. Além dos agentes sem dúvida patogênicos, aparecem com destaque altas taxas de comensais, indiscutíveis marcadores da influência da via fecal-oral na disseminação das infecções.

Não procuramos conhecer as implicações clínicas, pois nossa intervenção só teve nexo com diagnósticos parasitológicos. Contudo, alguns adoecimentos devem estar ocorrendo. Parece que tratamentos são improváveis e, lamentavelmente, a persistência de reinfecções é muito plausível, já que não percebemos perspectiva de mudanças de ordens pessoal e ou ambiental.

Cremos que dois tópicos merecem comentários específicos: a grande taxa de positividade referente ao Blastocystis hominis, como também o encontro de Cryptosporidium sp e Cyclospora cayetanensis.

Avaliações em múltiplos ambientes vêm registrando que o Blastocystis hominis comparece destacadamente. Esse protozoário não gerou, até agora, nenhum impacto se valorizada a saúde pública. Aparece abundantemente em diversos exames parasitológicos de fezes e continua sem definição a eventual capacidade patogênica dele, que sem dúvida deve refletir fácil transmissão pela via fecal-oral ${ }^{2} 35611$. Entre estes indígenas é bastante prevalente, aumentando preocupações e exigindo confiáveis explicações. É microrganismo à procura de doença e de devido posicionamento de caráter médico-sanitário.

A outra nota vincula-se ao encontro de Cryptosporidium sp e de Cyclospora cayetanensis. Tais parasitas ficaram durante muito tempo fora de conotações assistenciais em medicina humana. Despontaram quando imunodepressões passaram a ser mais reconhecidas e, entre elas, a atribuída ao vírus da imunodeficiência humana (HIV) causador da aids. De acordo com circunstâncias figuram como oportunistas. Talvez estivessem em cena há muito mais tempo e não eram procurados através do emprego de métodos laboratoriais adequados. Em nossa maneira de agir, agregamos a técnica de Kinyoun, eficaz para detectar os dois tipos de protozoários em questão, a técnicas tradicionais para execução de exames parasitológicos de fezes.

É perceptível que não estão incluídos o Strongyloides stercoralis nas análises, porque recebemos material em conservador que impede recorrer à extração de larvas, e devidamente o Enterobius vermicularis, cuja demonstração precisa de anal swab.

Se efetuados em outras ocasiões próximas os exames talvez fornecessem mais diagnósticos e isso é bem conhecido em Parasitologia; contudo, esta comunicação foi suficiente para destacar as más condições de higiene existentes na aldeia e fatos relacionados com Blastocystis hominis, Cryptosporidium sp e Cyclospora cayetanensis.

0 número de infectados por Hymenolepis nana denota também má situação sanitária, com participação de fecalismo; todavia, não houve especial especulação a respeito disso.

\section{AGRADECIMENTOS}

Para Mariana Quiroga, Melissa Mascheretti e Pasesa Quispe Torres pela cooperação prestada à realização desta avaliação parasitológica.

\section{REFERÊNCIAS}

1. Amato Neto V, Alarcón RSR, Gakiya E, Bezerra RC, Ferreira CS, Braz LMA Blastocistose: controvérsias e indefinições. Revista da Sociedade Brasileira de Medicina Tropical 36:515-517, 2003.

2. Amato Neto V, Alarcón RSR, Gakiya E, Ferreira CS, Bezerra RC, Santos AG. Elevada porcentagem de blastocistose em escolares de São Paulo, SP. Revista da Sociedade Brasileira de Medicina Tropical 37:354-356, 2004.

3. Amato Neto V, Corrêa LL. Exame parasitológico das fezes. 5ª edição. Editora Sarvier, São Paulo, 1991.

4. Clarke SC, Mclntyre M. Modified detergent Ziehl-Neelsen technique for the staining of Cyclospora cayetanensis. Journal of Clinical Pathology 49: 511-512, 1996.

5. Devera R. Blastocystis hominis: o enigma continua. Revista da Sociedade Brasileira de Medicina Tropical 31: 491-492, 1998.

6. Devera R. Blastocystis hominis: parasita intestinal pouco estudado no Brasil. Jornal Brasileiro de Medicina 76: 85-89, 1999.

7. Fontbonne A, Freese-de-Carvalho E, Acioli MD, Sá GA, Cesse EAP. Fatores de risco para poliparasitismo intestinal em uma comunidade indígena de Pernambuco, Brasil. Cadernos de Saúde Pública 17: 367-373, 2001. 
8. Lima MG, Koifman S, Scapulatempo IL, Peixoto M, Naomi S, Amaral MC. Fatores de risco para câncer de mama em mulheres indígenas Teréna de área rural, Estado do Mato Grosso do Sul, Brasil. Cadernos de Saúde Pública 17: 1537-1544, 2001

9. Morais MB, Alves GM, Fagundes-Neto U. Nutritional status of Terena indian children from Mato Grosso do Sul, Brazil: follow up of weight and height and current prevalence of anemia. Jornal de Pediatria (Rio de Janeiro) 81: 383-389, 2005.
10. Santos RV, Coimbra Jr CEA, Flowers NM, Silva JP. Intestinal parasitism in the Xavánte Indians, Central Brazil. Revista do Instituto de Medicina Tropical São Paulo 37: 145-148, 1995.

11. Velásquez V, Caldera R, Wong W, Cermeño G, Fuentes M, Blanco Y, Aponte M Devera R. Elevada prevalência de blastocistose em pacientes do Centro de Saúde de Soledad, Estado Anzoátegui, Venezuela. Revista da Sociedade Brasileira de Medicina Tropical 38: 356-357, 2005. 